\title{
A New UWB Pulse Design Method for Multiple Narrow-band Interference and Wide-band Interference Mitigation
}

\author{
$\mathrm{Xu} \mathrm{Lu}$, and Wen Chen \\ Department of Electronic Engineering \\ Shanghai Jiaotong University \\ Shanghai, P. R. China 200240 \\ Email: \{jarodlx; wenchen\}@ sjtu.edu.cn
}

\begin{abstract}
In this paper, we present a new method to generate ultra-wide band (UWB) pulses that not only meet the power spectral constraint of Federal Communications Commission (FCC), but also easily mitigate the multiple narrow-band interferences (NBI) distributed in the UWB band, which is allocated from $3.1 \mathrm{GHz}$ to $10.6 \mathrm{GHz}$. Especially, this method can be flexibly extended to suppress any wide-band interference located in the UWB band, even over GHz frequency.
\end{abstract}

Index Terms-UWB pulses, multiple narrow-band interference, eigenvalue decomposition.

\section{INTRODUCTION}

Due to the high data rate, low power consumption, and simple implementation, UWB systems show great potential in short range wireless communications [1],[2]. According to the Federal Communications Commission (FCC) regulations [3], the transmitted power level of UWB systems is limited to $41.3 \mathrm{dBm} / \mathrm{MHz}$ and can be spread over a huge bandwidth from $3.1 \mathrm{GHz}$ to $10.6 \mathrm{GHz}$. Therefore, the interference to the UWB systems from various narrow-band communication systems or wide-band communication systems such as GPS, IEEE 802.11WLANs, IEEE 802.16 WiMax becomes unavoidable [4].

The Gaussian monocycle pulses are commonly used in UWB impulse radio [5]-[8]. However, the Gaussian monocycle pulses need to be filtered to meet the FCC spectral mask. It turns out that the time duration of the corresponding pulses become too long, which severely reduces the data rate and capacity. In [9], a new pulse design method based on the eigenvalue decomposition algorithm is presented. And in [10], this method is extended to suppress the mutual interference between the narrow-band devices and the UWB systems.

Although the pulses designed in the aforementioned methods meet FCC spectral mask properly, and well suppress the single narrow-band interference, it is not suitable for the multiple interference situation. In this paper, we propose a new method to mitigate multiple narrow-band interferences located in the UWB band. Then we develop the method to the suppression of wide-band interference. By using our method, the multiple interferences among narrow-band systems, wide-band systems and UWB systems can be dramatically mitigated. So the coexisting problem of these devices can be well solved.

The rest of the paper is organized as follows. In Section II, firstly we introduce the eigenvalue decomposition method and then we give a short introduction to the single narrowband interference suppression proposed in [10]. In Section III, we present our new method to mitigate multiple narrow-band interference by dividing the UWB band into two sub-bands. In Section IV, we develop the method to the situation of wideband interference by dividing the UWB band into three subbands. Finally, in Section V, we conclude the paper.

\section{EIGENVALUE DECOMPOSITION ALGORITHM}

The algorithm for the UWB pulse design utilizing ideas of prolate spheroidal wave functions [11],[12] was first presented in [9]. Here the brief summary of this algorithm is introduced necessarily.

Firstly, a desired frequency mask $H(f)$ is given.

$$
H(f)=\left\{\begin{array}{cc}
1 & f_{L}<f<f_{H} \\
0 & \text { elsewhere }
\end{array}\right.
$$

where $f_{L}=3.1 \mathrm{GHz}, f_{H}=10.6 \mathrm{GHz}$. Simply we can get the inverse Fourier transform of $H(f)$.

$$
h(t)=2 f_{H} \operatorname{sinc}\left(2 f_{H} t\right)-2 f_{L} \operatorname{sinc}\left(2 f_{L} t\right)
$$

The mask is equivalent to a frequency response of a filter. So the UWB pulses $s(t)$ can be generated by filtering.

$$
\lambda s(t)=\int_{-\infty}^{\infty} s(\tau) h(t-\tau) d \tau
$$

where $\lambda$ is an attenuation factor, $h(t)$ is the inverse Fourier transform of $H(f)$.

We wish to design a pulse $p(t)$ that is time-limited.

$$
s(t)=0, \quad|t|>T_{m} / 2
$$

Clearly, $T_{m}$ is the time duration of UWB pulse. By sampling at a rate of $N$ samples per pulse period $T_{m},(2)$ can be expressed as follows:

$$
\lambda s[n]=\sum_{m=-N / 2}^{N / 2} s[m] h[n-m], n=-\frac{N}{2} \cdots \frac{N}{2}
$$




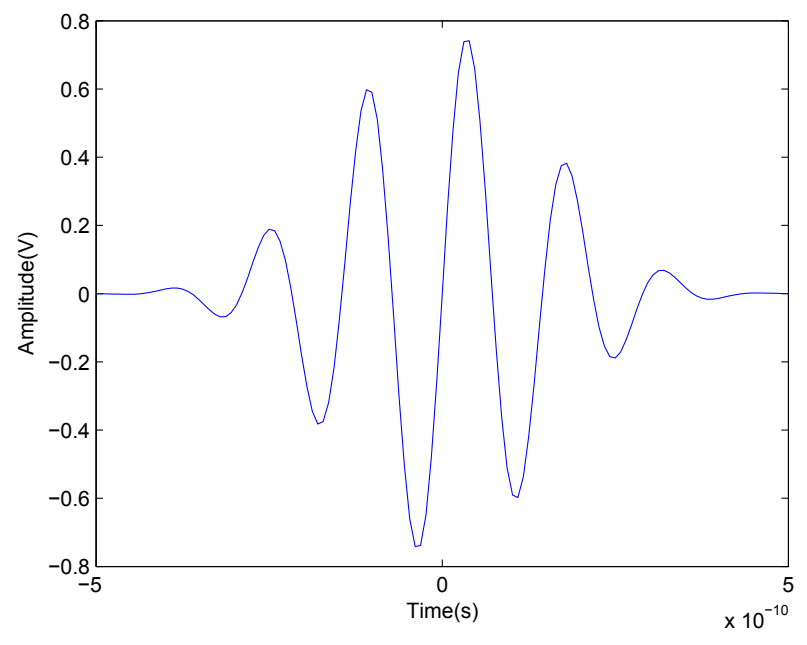

Fig. 1. UWB pulse obtained from eigenvalue decomposition algorithm

where $n$ and $m$ are integer values.

Equation (5) can be expressed in vector form as follows:

$$
\lambda \boldsymbol{s}=\boldsymbol{H} \boldsymbol{s}
$$

where vector $\boldsymbol{s}$ represents the discretized UWB pulse, $\boldsymbol{H}$ is a real Hermitian Toeplitz matrix,

$$
\boldsymbol{H}=\left(\begin{array}{cccc}
h[0] & h[-1] & \cdots & h[-N] \\
h[1] & h[0] & \cdots & h[-N+1] \\
\vdots & \vdots & & \vdots \\
h[N] & h[N-1] & \cdots & h[0]
\end{array}\right)
$$

Clearly, $\boldsymbol{s}$ is an eigenvector of $\boldsymbol{H}$, and $\lambda$ is the eigenvalue. Based on [9], only the eigenvectors corresponding to the largest eigenvalue should be taken as pulse designs and selected for implementation.

Fig. 1 shows an example of the UWB pulse generated by using the eigenvector corresponding to the largest eigenvalue of $\boldsymbol{H}$. Here, $N=128, T_{m}=1 \mathrm{~ns}$. The power spectral density (PSD) of the pulse is depicted in Figure2.

The frequency spectrum and power spectral density (PSD) of the UWB pulse can be calculated by

$$
\begin{gathered}
S(f)=\frac{T_{m}}{N} \sum_{n=-N / 2}^{N / 2} s[n] e^{-j 2 \pi f \frac{n T_{m}}{N}}, \\
P S D(f)=|S(f)|^{2} .
\end{gathered}
$$

In [10], the author discuss the mitigation of narrow-band interference that can be modeled as single frequency interference.

Suppose that the major power of the narrow-band interference is concentrated on its center frequency $f_{0}$. So the optimal scheme is to form a zero point of the PSD of UWB pulse at the frequency $f_{0}$. We can obtain the desired pulse as follows[10].

Firstly, partition the UWB band into two bands: $\left(f_{L}, f_{M}\right)$ and $\left(f_{N}, f_{H}\right)$. Note that $f_{N}>f_{M}$ is necessary.

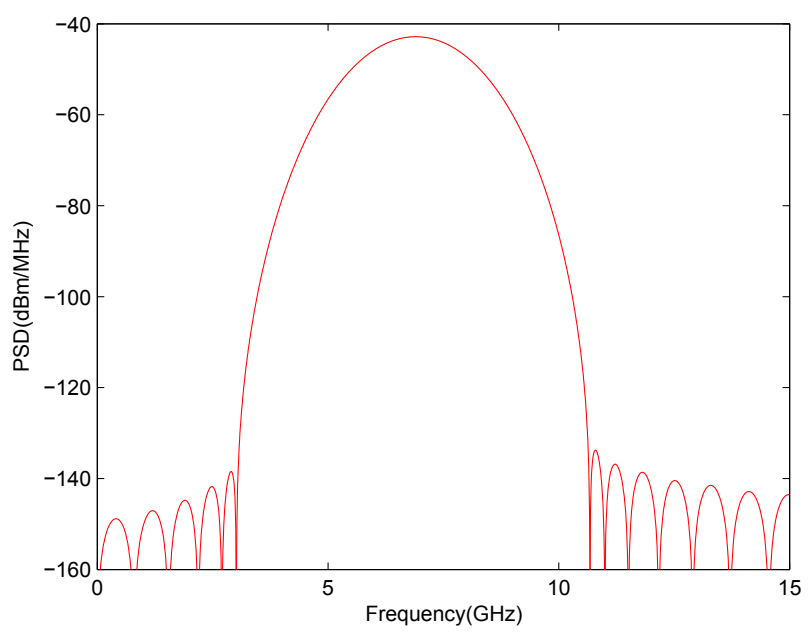

Fig. 2. PSD of generated UWB pulse

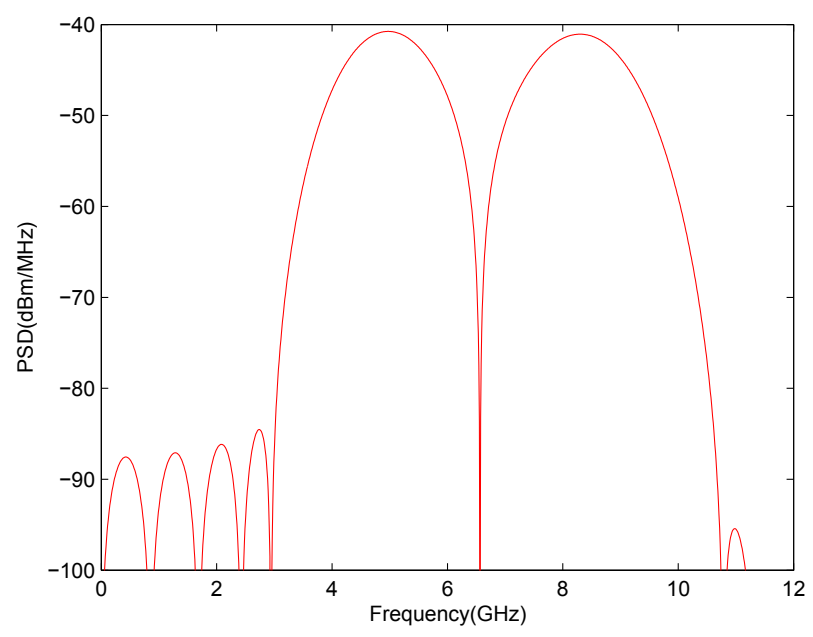

Fig. 3. PSD of the UWB pulse suppressing single NBI

Secondly, in each band, use the eigenvalue decomposition method to generate two sub-pulses $s_{1}(t)$ and $s_{2}(t)$. Meanwhile, adjust $f_{M}, f_{N}$ and pulse amplitude to make $s_{1}(t)$ and $s_{2}(t)$ not only meet the FCC spectral mask, but also let $S_{1}\left(f_{0}\right)=-S_{2}\left(f_{0}\right)$. Here, $S_{1}\left(f_{0}\right)$ and $S_{2}\left(f_{0}\right)$ denote the frequency spectrums of $s_{1}(t)$ and $s_{2}(t)$.

Thirdly, superpose the two sub-pulses to generate the desired UWB pulse. Clearly the PSD (power spectral density) of UWB pulse has a zero point at $f_{0}$. Fig. 3 shows the PSD of the UWB pulse suppressing single narrow-band interference.

\section{MULTIPLE NARROW-BAND INTERFERENCE MITIGATION}

In this section, we assume there are over three narrow-band interferences located in the UWB band. If we still use the method in [10], the number of sub-bands will be over four. After the simulation, we found that the lobe of the pulse becomes larger with the increment of the sub-band number. So we have to limit the number of the sub-band not more 


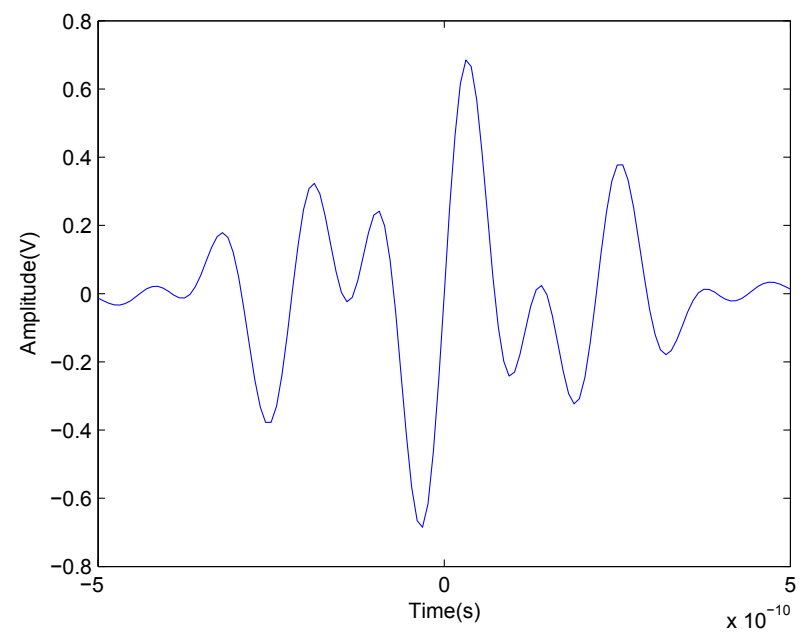

Fig. 4. combination of two sub-band pulses

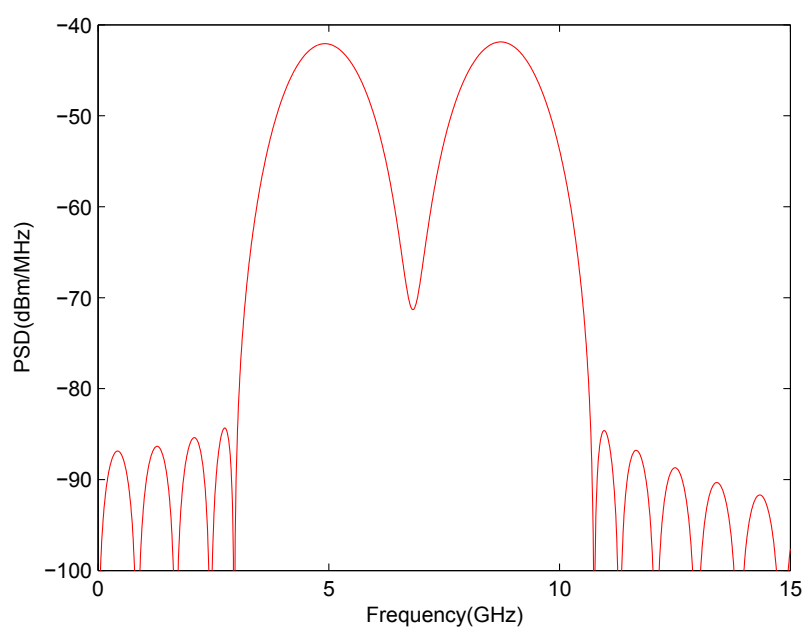

Fig. 5. PSD of combination of two sub-band pulses

than three. Under this limitation, we present our new method to deal with the multiple narrow-band interference situation. This method is simple, effective and easy-implemented. The main steps are as follows:

Step1. Partition the UWB band into two bands: $\left(f_{L}, f_{M}\right)$ and $\left(f_{M}, f_{H}\right)$, then use the eigenvalue decomposition method to generate two pulse $s_{1}(t), s_{2}(t)$ respectively. Without loss of generality, we suppose that the narrow-band interferences all locate in the lower band $\left(f_{L}, f_{M}\right)$. So at the receiver, $s_{1}(t)$ should be further processed to mitigate the narrow-band interferences.

Step2. We obtain the desired pulse from $s(t)=s_{1}(t)+$ $s_{2}(t)$. Fig.4 shows the waveform of $s_{3}(t)$, and Fig.5 depicts the PSD of the composed pulse, here we choose $f_{M}=6 \mathrm{GHz}$.

Step3. At the receiver, we use a doublet pulse consisting of two received pulses $s_{1}(t)$ separated from each other by $T_{g}$ time and with opposite amplitudes [13]. $T_{g}$ represents the

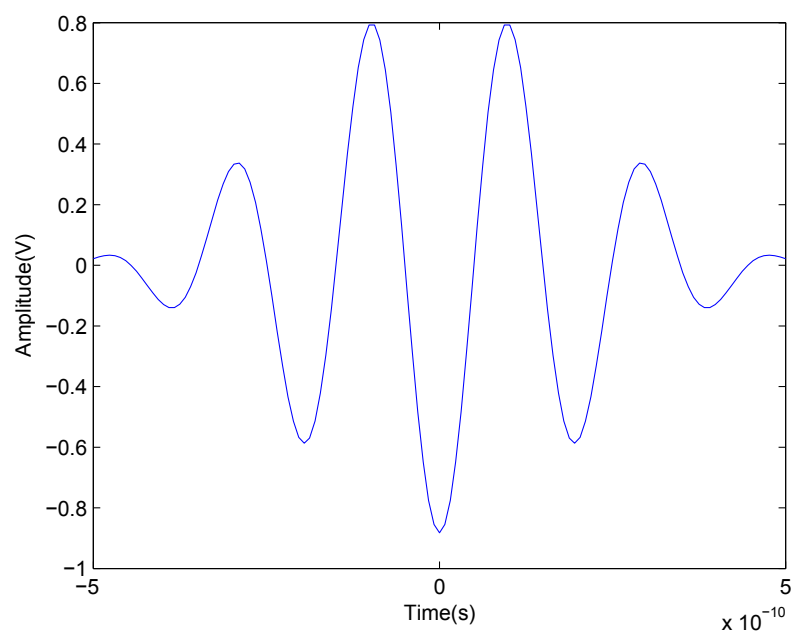

Fig. 6. waveform generated from lower band

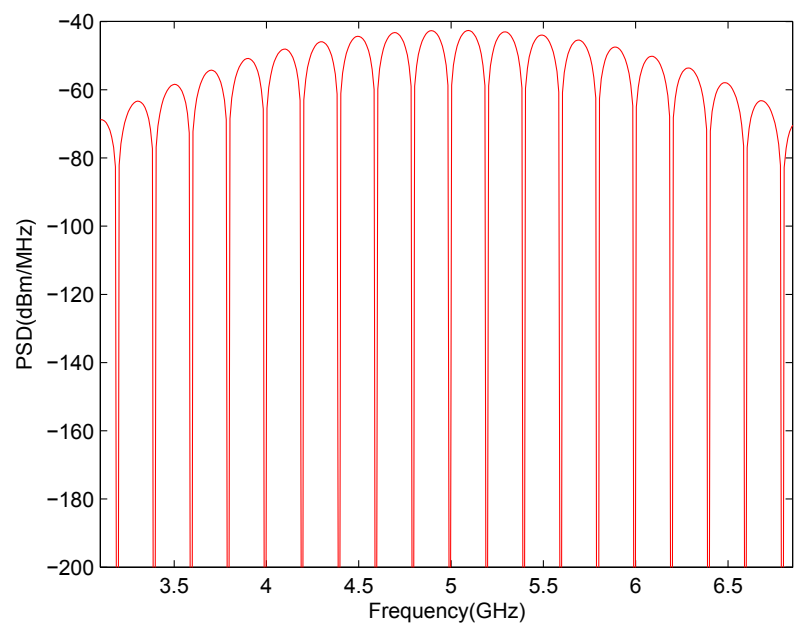

Fig. 7. PSD of lower band pulse

delay time. The processed pulse $s_{d}(t)$ can be expressed as:

$$
s_{d}(t)=\frac{1}{\sqrt{2}}\left(s_{1}(t)-s_{1}\left(t-T_{g}\right)\right)
$$

The spectral amplitude of such a pulse can be computed as:

$$
\left|S_{d}(f)\right|^{2}=2\left|S_{1}(f)\right|^{2} \sin ^{2}\left(\pi f T_{g}\right)
$$

Note that the spectrum has nulls at frequencies at $f=\frac{k}{T_{g}}$ for any integer $k$. By adjusting $T_{g}$, we can easily remove the interferences in the lower band. Fig.7 shows the spectral characteristic clearly.

\section{WIDE-BAND INTERFERENCE MITIGATION}

In this section, we extend our method to the wide-band interference mitigation. Commonly, the UWB pulse is applied in the WPAN (wireless personal area network). It is possible that the UWB systems coexist with several narrow-band devices and wide-band devices simultaneously. In this situation, 
the process is a little more complicated than two sub-bands mode discussed in the last section. We will divide the UWB band into three sub-bands.

We suppose that the interference has a bandwidth from $f_{I, L}$ to $f_{I, H}$, note that $\left(f_{I, L}, f_{I, H}\right)$ locates in the middle of the UWB band. The wide-band systems employ the multiple carries, the main transmitted power is concentrated on the carriers, so our main idea remains to generate nulls at each frequency of the carrier. The difference between this mode and two sub-bands mode is the delay time $T_{g}$ that could be longer according to the frequency intervals of the carriers. Therefore the wide-band interference can be dramatically mitigated. The steps of this mode are as follows:

Step1. Partition the UWB band into three bands: $\left(f_{L}, f_{I, L}\right)$, $\left(f_{I, L}, f_{I, H}\right)$, and $\left(f_{I, H}, f_{H}\right)$. Then use the eigenvalue decomposition method to generate three sub-pulses $s_{1}(t), s_{2}(t)$, and $s_{3}(t)$ in each sub-band respectively. Note that $s_{2}(t)$ is in the interference bandwidth. So at the receiver, we can not use it before further processing.

Step2. Compose a new pulse $s(t)$ with $s_{1}(t), s_{2}(t), s_{3}(t)$.

$$
s(t)=s_{1}(t)+s_{d}(t)+s_{3}(t)
$$

The spectrum of the new pulse is

$$
S(f)=S_{1}(f)+S_{2}(f)+S_{3}(f)
$$

Fig.8 shows the new combined pulse and Fig.9 describes its PSD.

Step3. Based on the idea proposed in the two sub-bands mode, the received pulse in middle band $s_{2}(t)$ is processed in the same way. The processed pulse $s_{d}(t)$ can be expressed as: $s_{d}(t)=\frac{1}{\sqrt{2}}\left(s_{2}(t)-s_{2}\left(t-T_{g}\right)\right)$. And the spectral amplitude of such a pulse can be computed as

$$
\left|S_{d}(f)\right|^{2}=2\left|S_{2}(f)\right|^{2} \sin ^{2}\left(\pi f T_{g}\right)
$$

Therefore, the spectrum has nulls at frequencies at $f=\frac{k}{T_{g}}$ for any integer $k$. Suppose the wide-band system utilizes $N$ carriers, and the frequency interval between two carriers is $F_{I}$. We set $T_{g}=\frac{1}{F_{I}}$. So we can remove all the interference on every carrier. Fig.10 shows the PSD of the processed pulse in the middle band.

\section{CONCLUSION}

In this paper, we present a new method to suppress the multiple narrow-band interference and wide-band interference located in the UWB band. The method includes two modes: Mode1 has two sub-bands, and Mode2 has three sub-bands. By using our new method, the generated UWB pulses have several advantages: Firstly, the pulse meets the FCC spectral mask; Secondly, it occupies a short time duration; Thirdly, the lobe of the obtained pulse is small. So it greatly solves the coexisting problem between UWB systems and multiple narrow-band systems or wide-band systems. Meanwhile because of its simple implementation, the design of UWB pulses can be applied extensively.

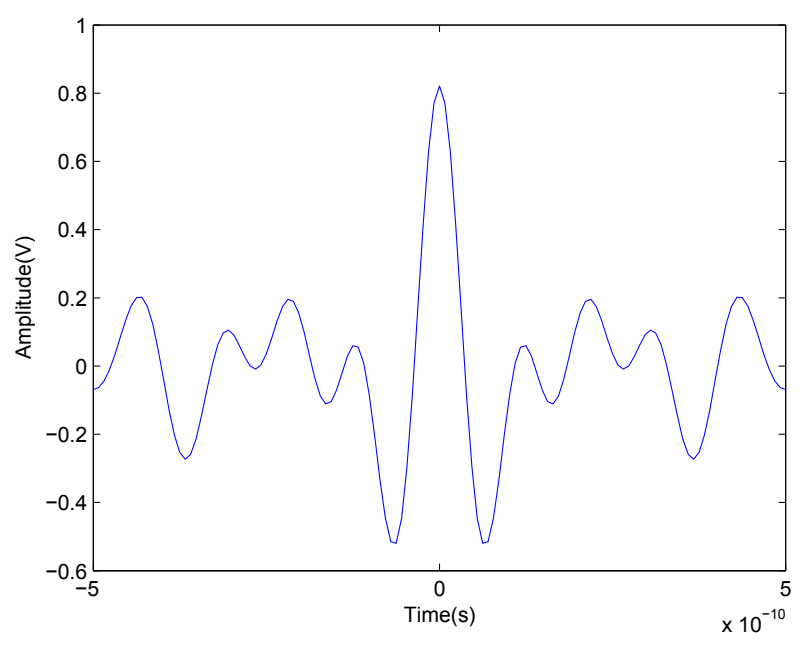

Fig. 8. combination of three sub-pulses

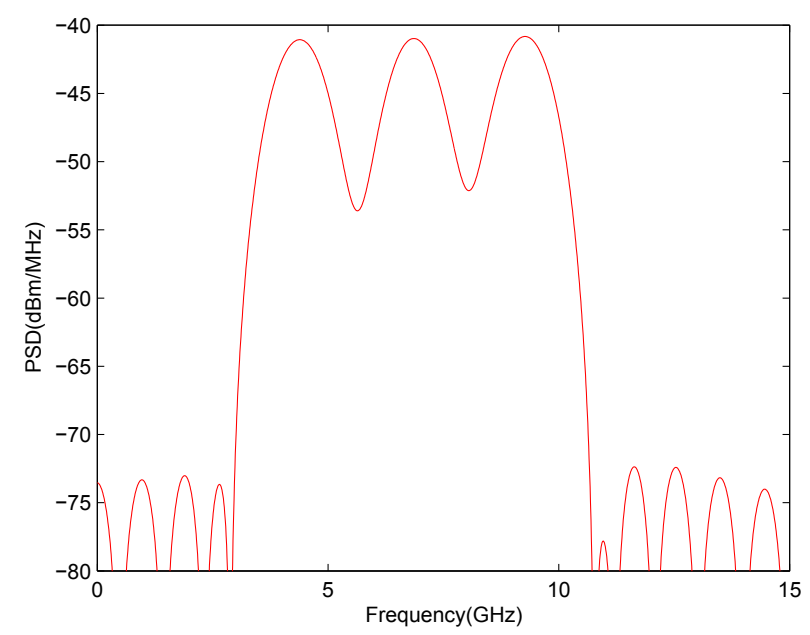

Fig. 9. PSD of the combination of three sub-pulses

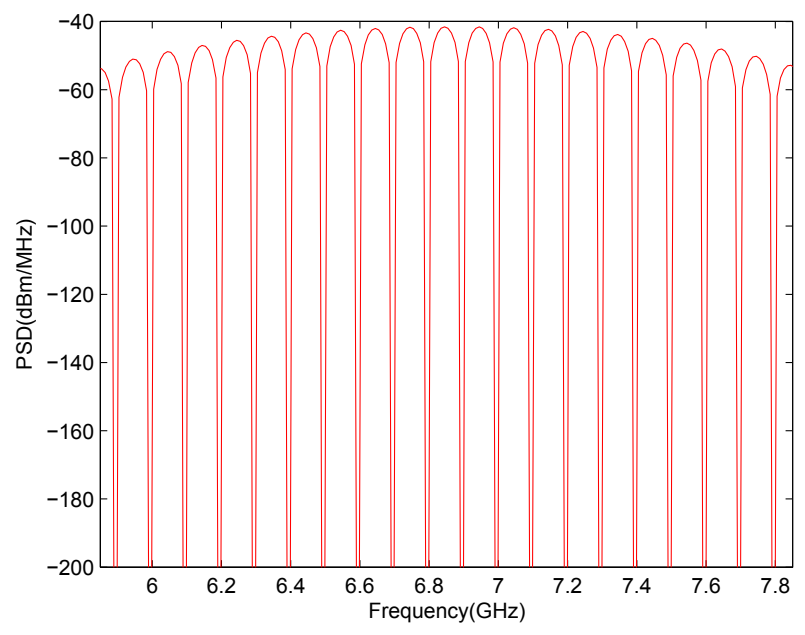

Fig. 10. PSD of received pulse in the middle band 


\section{ACKNOWLEDGMENT}

This work is supported by NSF China \#60672067, by NSF Shanghai \#06ZR14041, by Shanghai-Canada NRC \#06SN07112, by Cultivation Fund of the Key Scientific and Technical Innovation Project, Ministry of Education of China \#706022, by Program for New Century Excellent Talents in University \#NCET-06-0386, and by Pujiang Talent\#07pj4046.

\section{REFERENCES}

[1] M. Z. Win and R. A. Scholtz, "Impulse radio: How it works," IEEE Commun. Lett., vol. 2, pp. 36-38, Feb. 1998.

[2] L. Yang and G. B. Giannakis, "Ultra-wideband communications: An idea whose time has come," IEEE Signal Process. Mag., vol. 21, no.6, pp. 26-54, Nov. 2004.

[3] Fedral Communications Commission, New public safety applications and broadband Internet access among uses envisioned by FCC authorization of ultra-wideband technology Feb. 2002 [Online]. Available: http://www.fcc.gov/Bureaus/Engineering Technology/NewsReleases/2002/nret0203.html

[4] L. Yang and G. B. Giannakis, "Ultra-wideband multiple access: Unification and narrowband interference analysis," in proc.IEEE Conf. Ultra Wideband Systems Technologies, Nov.2003, pp. 320-324.

[5] S. S. Kolenchery, J. K. Townsend, and J. A. Freebersyser, "A novel inpulse radio network for tactical militray wireless communications," Millitary Communications Conf., Vol. 1, Boston, MA, Oct.1998, pp.59-65.

[6] "Revision of Part 15 the Commission's rules regarding ultra-wideband transmission systems," FCC, ET Docket 98-153, 2002.

[7] T. M. Cover and J. A. Thomas, Elements of Information Theory, New York: Wiley, 1991.

[8] J. Han and C. Nguyen, "A new ultra-wideband, ultra-short monocycle pulse generator with reduced ringing," IEEE Microwave Wireless Compon. Lett., vol. 12, pp.206-208, June 2002.

[9] Brent Parr, ByungLok Cho, et al, "A Novel Ultra-wideband Pulse Design Algorithm," IEEE Commun. Lett., vol. 7, no.5, pp. 36-38, May.2003.

[10] Zhendong Luo, Hong Gao, et al, "A New UWB Pulse Design Method for Narrowband Interference Suppression," IEEE Communications Society Globecom 2004.vol 1, no.5, pp. 3488-3492, April 2004.

[11] D. Slepian and H. O. Pollak, "Prolate spheroidal wave functions, Fourier analysis, and uncertainty-I," Bell Syst. Tech. J., vol.40, no.1, pp.43-46, Jan 1961.

[12] D. Slepian, "Prolate spheroidal wave functions, fourier analysis, and uncertainty-V: The discrete case," Bell Syst. Tech. J., vol.57, no.5, pp.1371-1430, May 1978.

[13] A. Taha., K. M. Chugg, "A theoretical study on the effects of interference UWB multiple access impulse radio Signals," Systems and Computers 2002 Conference Record of the Thirty-Sixth Asilomar Conference., Vol.1, pp.728-732, Nov 2002. 